\title{
CTGF directs fibroblast differentiation from human mesenchymal stem/stromal cells and defines connective tissue healing in a rodent injury model
}

\author{
Chang H. Lee, Bhranti Shah, Eduardo K. Moioli, and Jeremy J. Mao
}

Tissue Engineering and Regenerative Medicine Laboratory (TERML), College of Dental Medicine, Columbia University, New York, New York, USA.

\begin{abstract}
Fibroblasts are ubiquitous cells that demonstrate remarkable diversity. However, their origin and pathways of differentiation remain poorly defined. Here, we show that connective tissue growth factor (CTGF; also known as CCN2) is sufficient to induce human bone marrow mesenchymal stem/stromal cells (MSCs) to differentiate into fibroblasts. CTGF-stimulated MSCs lost their surface mesenchymal epitopes, expressed broad fibroblastic hallmarks, and increasingly synthesized collagen type I and tenacin-C. After fibroblastic commitment, the ability of MSCs to differentiate into nonfibroblastic lineages - including osteoblasts, chondrocytes, and adipocytes - was diminished. To address inherent heterogeneity in MSC culture, we established 18 single MSC-derived clones by limiting dilution. CTGF-treated MSCs were $\alpha$-SMA-, differentiating into $\alpha-S^{+} A^{+}$myofibroblasts only when stimulated subsequently with TGF- $\beta 1$, suggestive of stepwise processes of fibroblast commitment, fibrogenesis, and pathological fibrosis. In rats, in vivo microencapsulated delivery of CTGF prompted postnatal connective tissue to undergo fibrogenesis rather than ectopic mineralization. The knowledge that fibroblasts have a mesenchymal origin may enrich our understanding of organ fibrosis, cancer stroma, ectopic mineralization, scarring, and regeneration.
\end{abstract}

\section{Introduction}

Fibroblasts are ubiquitous cells that constitute the stroma of virtually all tissues. The conventional view of fibroblasts as no more than collagen-producing cells is being challenged by their interactions with immune and inflammatory cells and by their putative roles in cancer stroma as cancer-associated fibroblasts (1-5). Fibroblasts are remarkably diverse in different tissues across the body, and even within the same tissue (6). This remarkable diversity, along with a paucity of unique molecular markers for fibroblasts, prompted some of the literature to conveniently consider uncharacterized cells as fibroblasts, often based primarily on cell morphology (reviewed in refs. 7-9). A notable example is the important discovery of colony-forming unit fibroblasts in bone marrow (10), which were later demonstrated by the same investigators to differentiate into osteoblasts (11), nullifying their initial, misconceived qualification as fibroblasts. It is now clear that fractions of nonhematopoietic, fibroblast-like cells of bone marrow are stem/stromal cells that can undergo self-renewal for limited passages or differentiate into mesenchymal lineages, such as osteoblasts, adipocytes, chondrocytes, and myocytes $(7-9,12)$. In contrast to well-explored induction and differentiation pathways of other mesenchymal lineages, the origin of fibroblasts has remained poorly defined. A notion that has gained experimental support from multiple studies of organ fibrosis is that fibroblasts derive from epithelial or endothelial cells in a process known as endothelial- or epithelial-to-mesenchymal transition (EMT; refs. 13-15). Cell tracing in transgenic models showed transformation of endothelial or epithelial cells into fibroblast-like cells $(14,16)$.

Conflict of interest: The authors have declared that no conflict of interest exists. Citation for this article: J Clin Invest. 2010;120(9):3340-3349. doi:10.1172/JCI43230.
However, EMT does not account for all the fibroblasts that are present in organ fibrosis (17). Besides stromal cells, fibroblasts also act as parenchymal cells in several specialized connective tissues, such as ligaments and tendons. Compared to excessive fibroblasts and collagen biosynthesis in organ fibrosis in which fibroblasts are stromal cells, parenchymal fibroblastic tissues such as tendons and ligaments are recalcitrant to regeneration $(18,19)$. The poor innate healing capacity of parenchymal fibroblastic tissues is attributed to the paucity of fibroblasts as collagen-producing cells, among other factors $(18,19)$. Thus, EMT does not explain the origin of parenchymal fibroblasts, given the absence of either epithelial or endothelial cells in normal tendons or ligaments. Discovery of multipotent mesenchymal cells in tendons (20) provides initial and important clues for a longstanding hypothesis of the mesenchymal origin of fibroblasts $(8,9,21)$.

A putative mesenchymal origin of fibroblasts can be either bone marrow or connective tissue derived. Mesenchymal stem/stromal cells (MSCs) have been regarded as adherent cells to tissue culture polystyrene, are positive for CD73, CD90, and CD105 but negative for CD14 or CD11b, CD19, CD34, CD45, CD79a, and HLA-DR, and additionally have the capacity to differentiate into osteoblasts, adipocytes, and chondrocytes under permissive conditions (22). In bone marrow, $\mathrm{CD} 34^{+}$and $\mathrm{CD}_{4} 5^{+}$fibrocytes are regarded as a subpopulation of stem/progenitor cells with characteristics of hematopoietic stem cells, monocytes, and fibroblasts and can migrate to the periphery upon wounding (23-25). However, $\mathrm{CD}^{+} 4^{+}$and $\mathrm{CD} 45^{+}$fibrocytes account for less than $1 \%$ of total bone marrow cells (24) and are likely not involved in homeostasis of connective tissues throughout the body. Thus, the origin and differentiation pathways of fibroblasts responsible for homeostasis and repair of connective tissues upon insults such as trauma, can- 


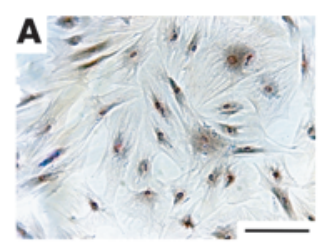

C
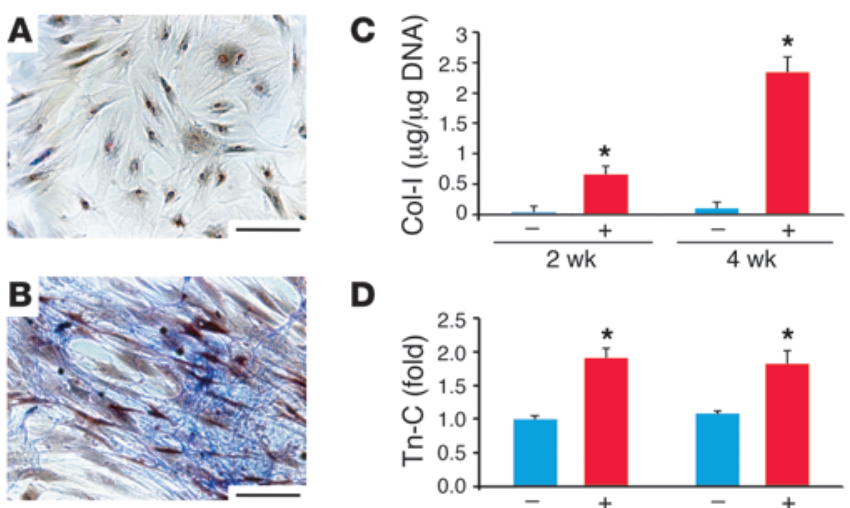

D
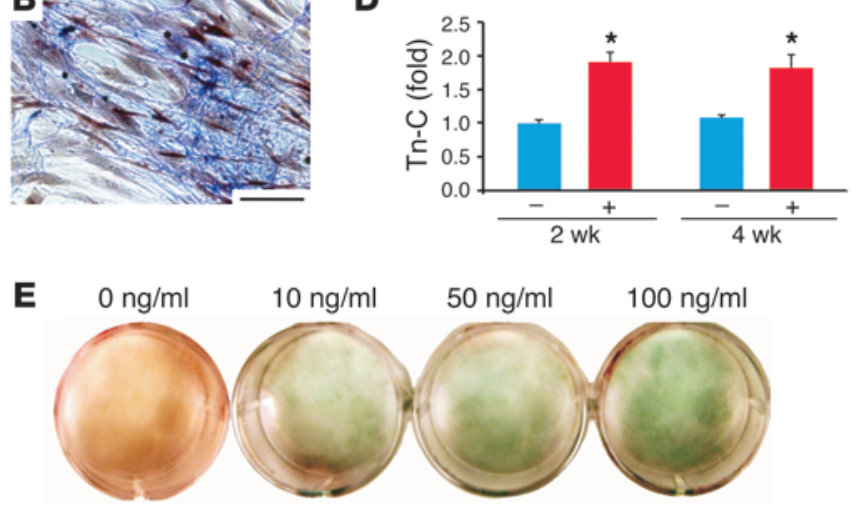
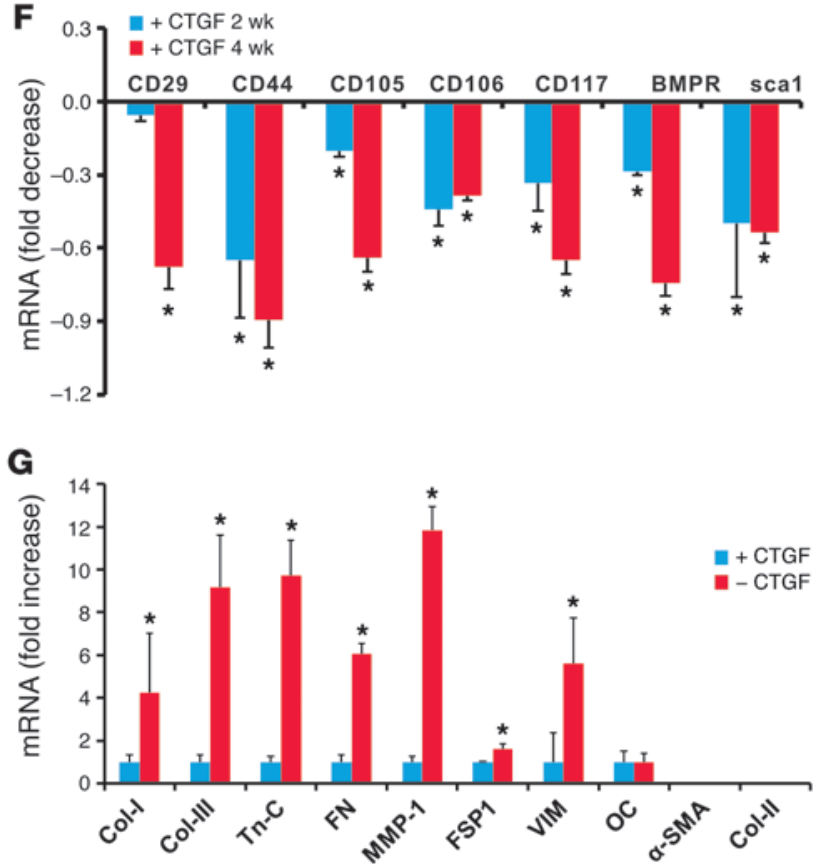

Figure 1

CTGF-mediated fibroblastic differentiation of MSCs. (A) Bone marrow MSCs were isolated and culture expanded. (B) CTGF treatment (100 ng/ml) prompted substantial collagen synthesis (Masson trichrome) compared with MSCs without CTGF treatment (A). (C and D) Col-I (C) and Tn-C (D) contents of CTGF-treated MSCs cells were significantly higher than MSCs without CTGF treatment at the 2- and 4-week time points $(n=5)$, as determined by ELISA. (E) Collagen deposition increased with increasing CTGF doses from 0 to $100 \mathrm{ng} / \mathrm{ml}$ (Goldner trichrome). (F) Levels of MSC surface epitopes were gradually attenuated, including CD29, CD44, CD105, CD106, CD117, BMPR1A, and Sca1 upon 2 and 4 weeks of CTGF treatment. (G) Concurrently, levels of fibroblastic markers gradually increased, including Col-I, Col-III, Tn-C, fibronectin (FN), MMP-1, FSP1, and vimentin (VIM) upon 4 weeks of CTGF treatment. The chondrogenic marker Col-II and myofibroblastic marker $\alpha$-SMA were undetectable, whereas the osteogenic marker osteocalcin (OC) was minimally expressed (G). Scale bars: $100 \mu \mathrm{m}$. Data represent mean $\pm \mathrm{SD}$. ${ }^{*} P<0.05$.

cer, and infection remain poorly elucidated. Our understanding of a given cell type is rarely comprehensive unless its origins and differentiation pathways are illustrated. Here, we induced MSCs from both appendicular and calvarial bone marrows to differentiate into cells that were reminiscent of fibroblasts. Connective tissue growth factor (CTGF; also known as CCN2), a $36-38 \mathrm{kDa}$, cysteine-rich protein of the CCN family (26), sufficiently prompted MSCs to differentiate into fibroblasts. Two-thirds of clonal progenies of human bone marrow-adherent cells differentiated into fibroblasts upon CTGF stimulation, as well as into osteogenic, chondrogenic, and adipogenic lineages under permissive conditions. Importantly, CTGF-treated MSCs were $\alpha$-SMA-; only when further stimulated with TGF- $\beta 1$ did they differentiate into $\alpha$-SMA ${ }^{+}$, myofibroblast-like cells that significantly contracted collagen gel in vitro. Control-released CTGF from biocompatible microspheres restored morphogenesis and microscopic characteristics of a mesenchymal/fibrogenic calvarial suture in vivo that otherwise was destined to undergo ectopic mineralization. Collectively, the mesenchymal origin of fibroblasts may have implications in our understanding of cancer stroma, organ fibrosis, ectopic mineralization, and scarring versus regeneration.

\section{Results}

CTGF differentiates MSCs into fibroblastic cells. Nonhematopoietic, mononuclear, adherent cells were isolated from multiple adult primary human bone marrow samples (Figure 1A), as we described previously $(12,27)$. Exposure of $100 \mathrm{ng} / \mathrm{ml} \mathrm{recombi-}$ nant human CTGF induced remarkable collagen synthesis by 4 weeks (Figure 1B), in comparison to MSCs without CTGF treatment (Figure 1A). CTGF was selected because of its roles in promoting fibroblast proliferation $(26,28,29)$. As determined quantitatively by ELISA, collagen type I (Col-I) and tenacin-C (Tn-C) synthesis by CTGF-treated MSCs was significantly greater at 2 and 4 weeks than that in MSCs without CTGF treatment (Figure 1, C and D). CTGF at $10 \mathrm{ng} / \mathrm{ml}$ was sufficient to stimulate collagen synthesis of MSCs, although 50 and $100 \mathrm{ng} / \mathrm{ml}$ were apparently more potent (Figure 1E), in comparison to MSCs without CTGF (Figure 1E, left). A broad array of MSC surface epitopes (27), including CD29, CD44, CD105, CD106, CD117, bone morphogenetic protein receptor type IA (BMPR1A), and Sca1, showed steady decreases by real-time PCR over the observed 2 and 4 weeks following CTGF treatment $(P<0.01$; Figure 1F). Concurrently, a cascade of fibroblastic hallmarks, including Col-I, Col-III, Tn-C, fibronectin, MMP-1, fibroblast-specific protein-1 (FSP1), and vimentin, drastically increased upon CTGF stimulation (Figure 1G). A late-stage osteogenic marker, osteocalcin, was minimally expressed, and a chondrogenic marker, Col-II, was undetectable by real-time PCR, which suggests that CTGF-treated MSCs were not differentiating into either osteoblasts or chondrocytes, 2 common mesenchymal lineages. Importantly, CTGF-stimulated MSCs remained $\alpha$-SMA- (Figure $1 \mathrm{G})$, which we further substantiated (see below). 

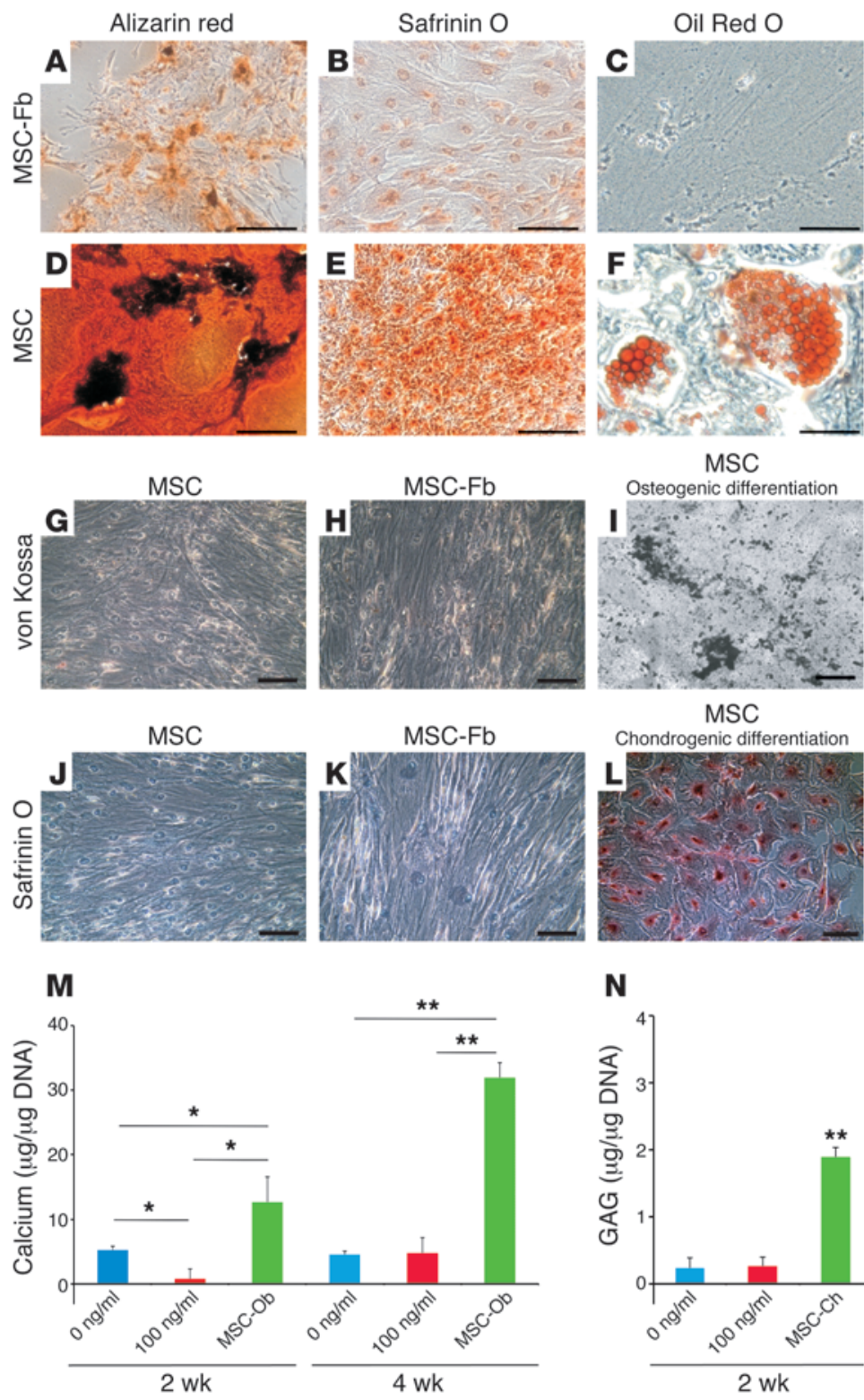

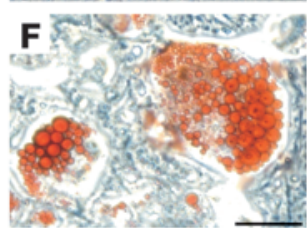

MSC

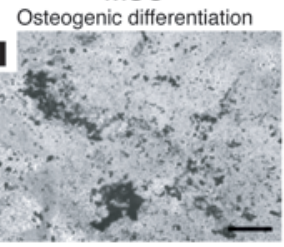

MSC

Chondrogenic differentiation

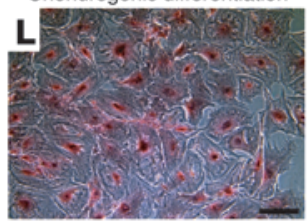

$\mathbf{N}$

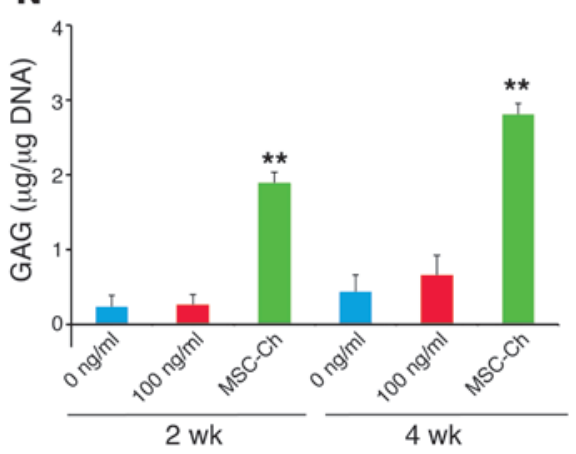

\section{Figure 2}

CTGF-derived fibroblasts are a stable population. (A-F) MSC-derived fibroblasts (MSC-Fb) by CTGF treatment for 4 weeks showed minimal capacity to further differentiate into osteoblasts $(\mathbf{A})$, chondrocytes (B), or adipocytes (C). In contrast, native MSCs, without CTGF treatment, readily differentiated into osteoblasts (D), chondrocytes (E), and adipocytes (F). (G-I) von Kossa staining was negative in CTGF-treated MSCs $(\mathbf{H})$, just as MSCs without CTGF treatment (G). (I) In contrast, MSCs subjected to osteogenic stimulation readily differentiated into osteogenic cells that elaborated minerals. (J-L) Safranin $O$ staining was negative in CTGF-treated MSCs (K), just as in MSCs without CTGF treatment (J). (L) In contrast, MSCs subjected to chondrogenic stimulation readily differentiated into chondrogenic cells that were safranin O positive. (M) Quantitatively, MSCs under osteogenic stimulation (MSC-Ob) elaborated significantly more calcium than did MSCs with or without CTGF treatment $(n=5)$. (N) In parallel, MSCs under chondrogenic stimulation (MSC-Ch) produced significantly more glycosaminoglycans (GAG) than MSCs with or without CTGF treatment $(n=5)$. Scale bars: $100 \mu \mathrm{m}(\mathbf{A}, \mathbf{B}, \mathbf{D}, \mathbf{E}$, and $\mathbf{G}-\mathbf{L})$; $50 \mu \mathrm{m}(\mathbf{C}$ and $\mathbf{F})$. Data represent mean \pm SD. ${ }^{*} P<0.05 ;{ }^{*} P<0.01$.
Attenuated ability of CTGF-stimulated MSCs to differentiate into nonfibroblastic lineages. Upon 4 weeks of CTGF treatment $(100 \mathrm{ng} / \mathrm{ml})$, MSCs showed diminished ability to differentiate into osteogenic cells, chondrogenic cells, and adipogenic cells, in contrast to CTGFfree culture of MSCs, which readily differentiated into osteoblasts (alizarin red positive), chondrocytes (safranin O positive), and adipocytes (Oil Red O positive) under corresponding permissive conditions (Figure 2, A-F, and see Methods). Furthermore, CTGF-treated cells were neither osteogenic nor chondrogenic. von Kossa staining was negative in CTGF-treated MSCs, just as in MSCs without CTGF treatment (Figure 2, G and H). In contrast, MSCs subjected to osteogenic stimulation readily differentiated into osteogenic cells that elaborated minerals (Figure 2I). Safranin O staining was negative in CTGF-treated MSCs, just as in MSCs without CTGF treatment (Figure 2, J and K). In contrast, MSCs subjected to chondrogenic stimulation readily differentiated into chondrogenic cells that were safranin O positive (Figure 2L). Quantitatively, MSCs under osteogenic stimulation elaborated significantly more calcium than did MSCs with or without CTGF treatment ( $n=5$; Figure 2M). In parallel, MSCs under chondrogenic stimulation produced significantly more glycosaminoglycans than did MSCs with or without CTGF treatment $(n=5$; Figure $2 \mathrm{~N})$.

Clonal progenies of MSCs differentiate into mesenchymal lineages. To address the notion that MSC-derived fibroblastic cells arise from fibroblasts in typical heterogeneous MSC culture, we isolated clones from MSCs and determined their lineage commitment and differentiation potential. Table 1 provides frequencies of clonal progenies of human bone marrow MSCs and the differentiation capacity of isolated clonal progenies. In total, 18 single cell-derived clones were established from 43 plated wells (about $42 \%$ ) by limiting dilution $(10,30)$. Of the 18 isolated clones, 12 clonal progenies (approximately 67\%) differentiated into all of fibroblastic, osteogenic, chondrogenic, and adipogenic cells, whereas 2 clonal progenies (approximately 11\%) 
Table 1

Clonal progenies of human MSCs and their differentiation potential

$\begin{array}{lcc}\text { Clones } & \boldsymbol{n} & \text { Differentiation potential (\%) } \\ \text { Yielded progenies } & 18 & 100 \% \\ \text { Quatropotent }{ }^{\mathrm{A}} & 12 & \sim 67 \% \\ \text { Tripotent }^{\mathrm{B}} & 2 & \sim 11 \% \\ \text { No differentiation } & 4 & \sim 22 \%\end{array}$

In total, 43 bone marrow-adherent cells were plated. ${ }^{A}$ Differentiated into fibroblasts, osteoblasts, chondrocytes, and adipocytes. ${ }^{B}$ Differentiated into fibroblasts, osteoblasts, and chondrocytes.

differentiated into fibroblastic, osteogenic, and chondrogenic cells, but not into adipogenic cells. The remaining 4 clonal progenies (approximately 22\%) failed to differentiate into any of fibroblastic, osteogenic, chondrogenic, or adipogenic cells (Table 1). Among the 3 clones shown here (B7, B12, and E3), B7 and B12 readily differentiated into fibroblast-like cells that elaborated collagen (Figure 3, A-C); osteogenic cells that produced alkaline phosphatase and minerals (Figure 3, D-F); adipogenic cells that were Oil Red O positive, with the notable exception of E3 (Figure 3, G-I); and chondrogenic cells that were safranin $\mathrm{O}$ positive (Figure $3, \mathrm{~J}-\mathrm{L}$ ). These clonal data suggest that bone marrow-derived MSCs indeed contain multipotent cells that are not end-stage fibroblasts, but can differentiate into multiple mesenchymal lineages, including fibroblasts. Of equal importance was the failure of approximately $22 \%$ of clones to differentiate into any of fibroblastic, osteogenic, chondrogenic, or adipogenic cells, which suggests that these cells are likely terminally differentiated bone marrow cells such as fibroblasts, osteoblasts, or other mononucleated cells.
CTGF-derived fibroblasts are $\alpha$-SMA-.$\alpha$-SMA is a pivotal hallmark of myofibroblasts. Gain of $\alpha$-SMA by myofibroblasts has profound significance in dermal wound healing, cancer stroma, and organ fibrosis $(1,2,4,5)$. Given that $\alpha$-SMA was undetectable in CTGFstimulated MSCs (Figure 1G), we analyzed $\alpha$-SMA expression with a known stimulant for myofibroblast phenotype, TGF- $\beta 1$. We first determined that $\alpha$-SMA was virtually absent in MSC culture (Figure 4A). Remarkably, TGF- $\beta 1$-treated MSCs only expressed modest $\alpha$-SMA (Figure 4B), which suggests that TGF- $\beta 1$ alone is not sufficient for MSCs to acquire myofibroblastic phenotype. Interestingly, little $\alpha$-SMA was present in MSC-derived fibroblasts (Figure 4C), which indicates that CTGF-treated MSCs, just like native MSCs, do not readily acquire myofibroblastic phenotype without TGF- $\beta 1$. Strikingly, TGF- $\beta 1$ treatment of MSC-derived fibroblastic cells readily expressed $\alpha$-SMA (Figure 4D), suggestive of a stepwise process toward fibroblastic commitment (i.e., CTGF) and gain of myofibroblastic phenotype (i.e., CTGF followed by TGF- $\beta 1$ ). Flow cytometry confirmed a general absence of $\alpha$-SMA of native MSCs (Figure 4E) or MSC-derived fibroblasts (Figure 4G). Strikingly, only approximately $2 \%$ of TGF- $\beta 1$-treated MSCs, with no prior CTGF treatment, gained an $\alpha$-SMA phenotype (Figure $4 \mathrm{~F})$. Importantly, approximately $32 \%$ of TGF- $\beta 1$-treated, MSCderived fibroblastic cells gained an $\alpha$-SMA phenotype (Figure 4H). In an established collagen gel contraction model, we found that MSCs with sequential exposure to CTGF (4 weeks) and TGF- $\beta 1$ (1 week) yielded the most significant contractility (Figure 4I), compared with CTGF stimulation alone (Figure $4 \mathrm{~J}$ ) or with TGF- $\beta 1$ stimulation alone (Figure 4K). Native MSCs, without either TGF- $\beta 1$ or CTGF stimulation, were least capable of contracting collagen gels (Figure 4L). Quantitative data confirmed that sequential stimulation of MSCs by CTGF and TGF- $\beta 1$ yielded the most significant collagen contraction (Figure 4M).

\section{Figure 3}

Clonal progenies of MSCs differentiate into multiple mesenchymal lineages. To address the heterogeneity of typical culture of MSCs by adherence to cell culture polystyrene, we established a total of 18 single cell-derived clones from 43 plated wells (approximately $42 \%$ ) by limiting dilution. Of the 18 isolated clones, 12 clonal progenies (about 67\%) differentiated into all of fibroblastic, osteogenic, chondrogenic, and adipogenic cells, whereas a total of 2 clonal progenies (about 11\%) differentiated into fibroblastic, osteogenic, and chondrogenic cells, but not into adipogenic cells. The remaining 4 clonal progenies (about $22 \%$ ) failed to differentiate into any of fibroblastic, osteogenic, chondrogenic, or adipogenic cells. (A-L) Clones, B7, B12, and E3 are shown. All 3 tested clones readily differentiated into fibroblast-like cells that elaborated collagen (A-C); osteogenic cells that produced alkaline phosphatase and minerals (D-F); adipogenic cells that were Oil Red O positive (G and $\mathbf{H}$ ), with a notable exception of E3 (I); and chondrogenic cells that were safranin $O$ positive $(\mathbf{J}-\mathbf{L})$. Scale bars: $100 \mu \mathrm{m}$ (A-F and $\mathbf{J}-\mathbf{L}) ; 50 \mu \mathrm{m}(\mathbf{G}-\mathbf{I})$.
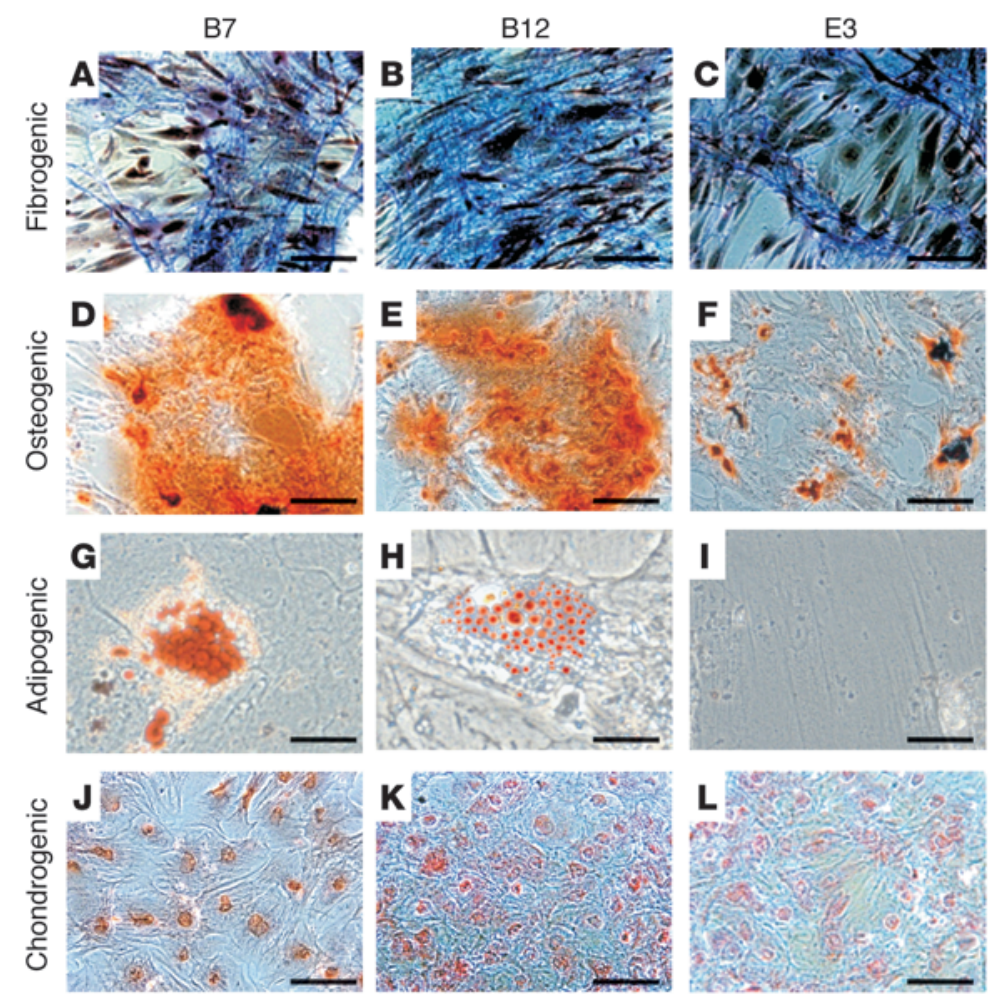


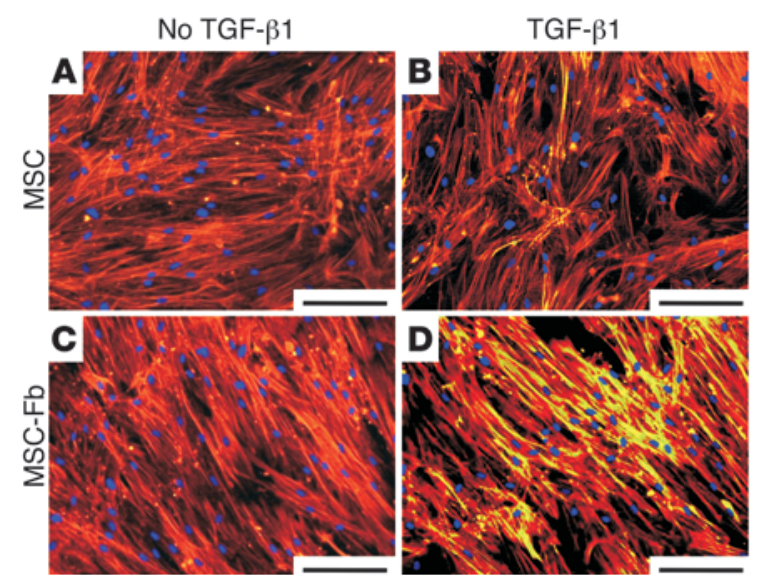

DAPI/F-actin/
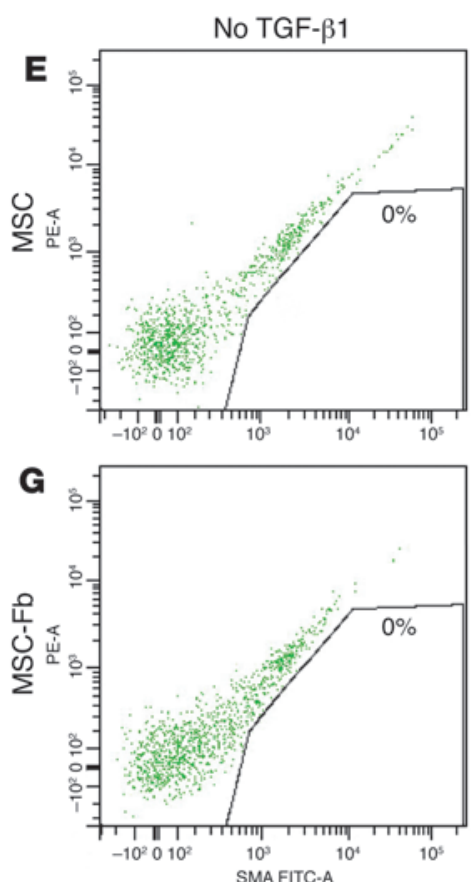
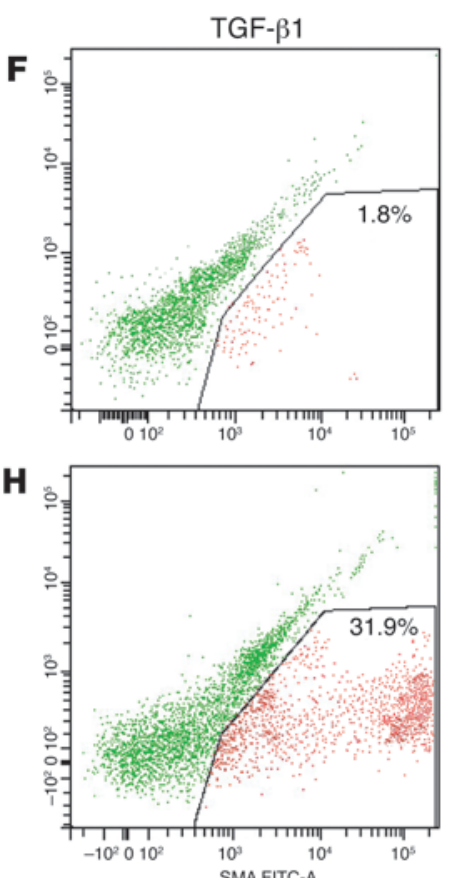
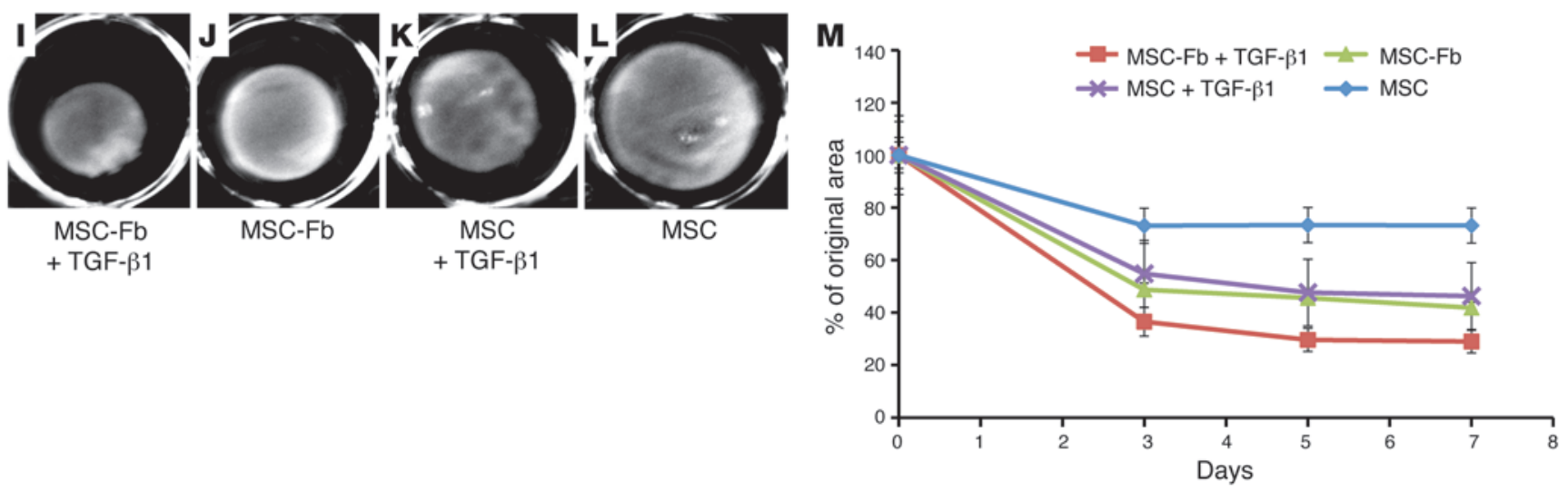

Figure 4

Myofibroblastic differentiation of MSC-derived fibroblastic cells by TGF- $\beta 1$. (A-D) Native MSCs (A) or MSC-derived fibroblasts by CTGF treatment $(\mathbf{C})$ expressed little $\alpha$-SMA. Upon TGF- $\beta 1$ treatment, native MSCs still expressed little $\alpha$-SMA (B), but MSC-derived fibroblasts readily expressed $\alpha-S_{M A}+$ microfilaments (D). (E-H) Flow cytometry confirmed the virtual absence of $\alpha$-SMA expression in MSCs (E) or MSC-derived fibroblasts (G). In contrast, $31.9 \%$ of MSC-derived fibroblasts $(\mathbf{H})$, but only $1.8 \%$ of native MSCs (F), gained $\alpha$-SMA phenotype after TGF- $\beta 1$ stimulation. (I-L) Collagen gel contraction assay showed that MSCs with sequential administration of CTGF (4 weeks) and TGF- $\beta 1$ (1 week) yielded the most significant contraction (I), compared with moderate contraction upon CTGF stimulation alone (J) or TGF- $\beta 1$ stimulation alone of native MSCs (K). MSCs without either CTGF or TGF- $\beta 1$ stimulation yielded the least contraction (L). (M) Quantitatively, sequential stimulation of MSCs by CTGF and TGF- $\beta 1$ yielded the most significant collagen gel contraction $(P<0.05)$. Scale bars: $100 \mu \mathrm{m}$. Data represent mean \pm SD.

CTGF favors fibrogenesis rather than ectopic mineralization in connective tissue healing. Ectopic mineralization is a poorly understood biologic process that occurs in disorders such as cardiac valve calcification, vascular arthrosclerosis, tendinopathy, craniosynostosis, calcified dermal healing, mineralization, and around implanted medical devices (31-33). Here, we used craniosynostosis as an in vivo model to test whether CTGF determines the outcome of connective tissue healing, given its above-described in vitro potency in defining a fibrogenic fate of multipotent mesenchymal cells. Craniosynostosis is one of the most common craniofacial anomalies (approximately 1 in 2,500 live human births), characterized by premature ectopic mineralization of mesenchymal/fibroblastic calvarial sutures that leads to craniofacial disfiguration in infants. Current surgical treatment is highly traumatic, frequently involving craniotomy in order to remove synostosed calvarial sutures and reshape multiple calvarial bones in early childhood. We adopted our previously developed control-release approach to potentiate the bioactivity of CTGF in vivo by microencapsulation, given the limited effectiveness of injected proteins or peptides without coating by rapid denature and diffusion $(34,35)$. Upon surgical removal of a synostosed calvarial suture in an established rat craniosynostosis model (36), the outcome of tissue repair was the predicted synostosis recurrence (Figure 5A) with complete obliteration of ectopic bone (Figure 5, E and G), similar to the synostosis recurrence previously 

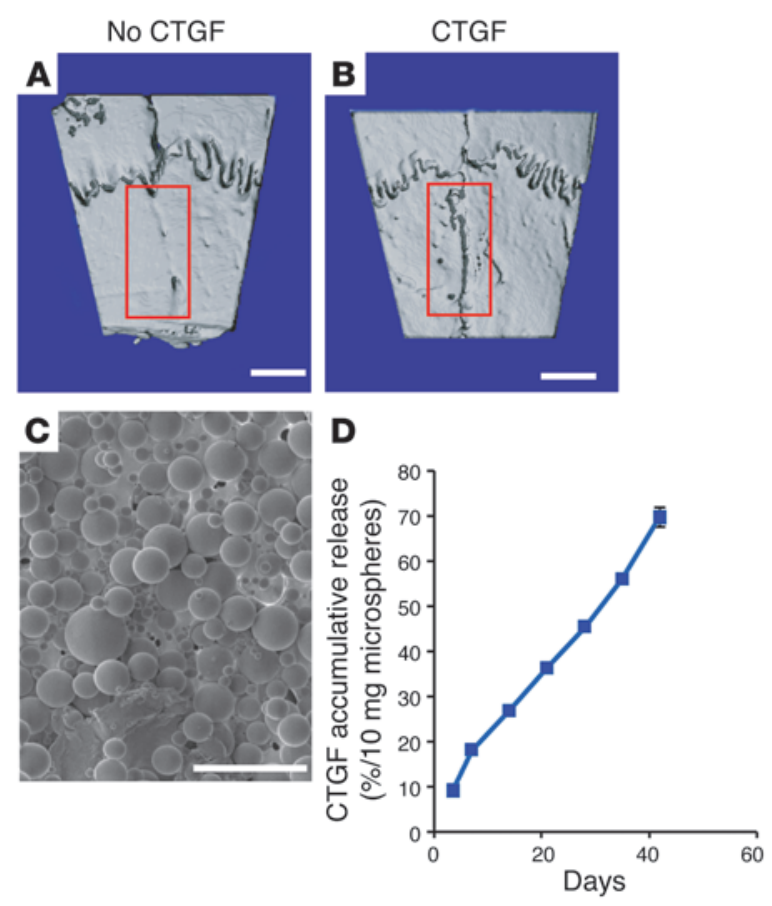
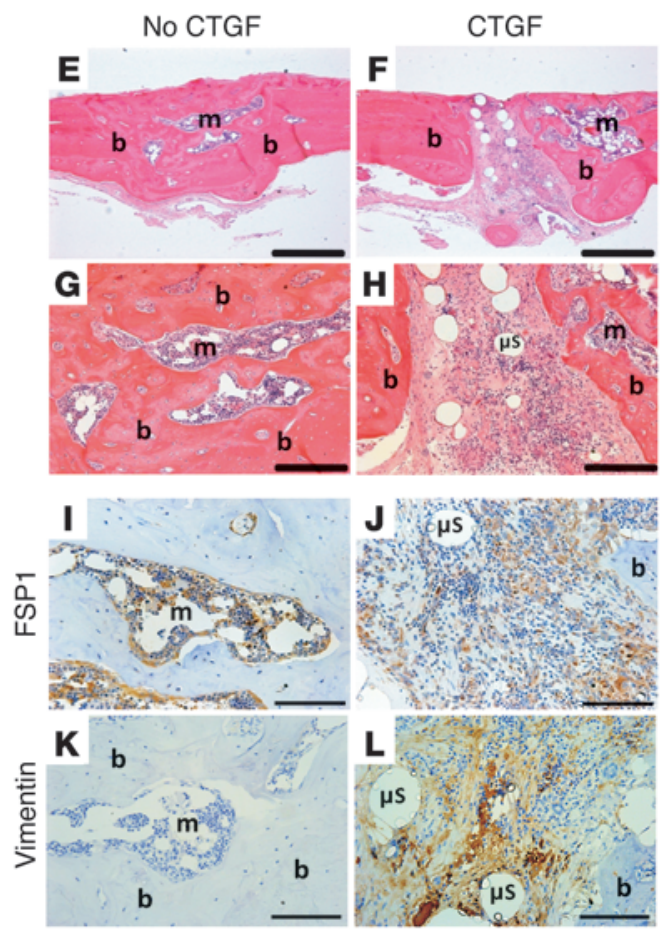

\section{Figure 5}

CTGF induced fibrogenesis instead of ectopic mineralization in vivo. (A and B) Representative 3D-reconstructed $\mu$ CT images after resection of a synostosed calvarial suture. Without CTGF, ectopic mineralization (boxed region) was readily observed (A), whereas anatomic morphology was restored in the absence of ectopic mineralization upon controlled release of CTGF (B). (C and D) CTGF-encapsulated PLGA microspheres were $120 \pm 64 \mu \mathrm{m}$ in diameter per scanning EM (C) and showed sustained release up to 6 weeks in vitro (D) $(n=6)$. (E-H) H\&E staining showed that microscopic morphology of the calvarial suture was restored upon controlled release of CTGF (F and $\mathbf{H})$, compared with ectopic mineralization without CTGF delivery ( $E$ and $\mathbf{G})$. Some CTGF-encapsulated microspheres $(\mu \mathrm{s})$ remained present at 4 postoperative weeks $(\mathbf{F}$ and $\mathbf{H})$. (I-L) Abundant expression of FSP1 (J) and vimentin (L) indicated the presence of fibroblast-like cells in regenerated calvarial suture; without CTGF delivery $(\mathbf{I}$ and $\mathbf{K})$, expression was restricted to the marrow $(\mathrm{m})$ of obliterated bone (b). Scale bars: $1 \mathrm{~mm}(\mathbf{A}$ and $\mathbf{B}) ; 500 \mu \mathrm{m}(\mathbf{C}, \mathbf{E}$, and F); $200 \mu \mathrm{m}$ $(\mathbf{G}-\mathbf{L})$. Data represent mean \pm SD.

described in craniosynostosis patients (36). Strikingly, controlreleased CTGF (Figure 5, C and D) alone prompted fibrogenesis and restored the morphogenesis of an anatomic structure reminiscent of a native calvarial suture (Figure 5B and ref. 36). The in vitro release profile of microencapsulated CTGF up to the tested 42 days is shown in Figure 5D. CTGF delivery by controlled release, besides reinstating the anatomic contour of the calvarial suture (Figure 5B), further restored the suture's microscopic characteristics with fibroblast-like cells in the soft tissue interface between mineralized bone (Figure 5, F and $\mathrm{H}$ ). The presence of microspheres in a bioengineered soft tissue interface (Figure 5, $\mathrm{F}$ and $\mathrm{H}$ ) indicated that microencapsulated CTGF was continuously released, given that release of encapsulated peptides and proteins only takes place upon microsphere degradation (34). Importantly, control-released CTGF induced abundant FSP1 and vimentin expression in the restored calvarial suture (Figure 5, J and L), in comparison to the presence of FSP $1^{+}$cells in the marrow of obliterated bone (Figure 5I) and the general absence of vimentin without CTGF delivery (Figure 5K), which suggests that CTGF favors fibrogenesis in calvarial healing rather than ectopic mineralization.

Ex vivo calvaria culture using a previously established model (37) revealed substantially similar findings to the above-described in vivo craniosynostosis model. At P10, a patent calvarial suture was characterized by the presence of mesenchymal/fibroblastic tissue between 2 bone formation fronts (Supplemental Figure 1A; supplemental material available online with this article; doi:10.1172/ JCI43230DS1) and by the expression of FSP1 and vimentin (Supplemental Figure 1, D and G). Without CTGF delivery, the calvarial suture readily underwent synostosis by P35 (i.e., P10 calvaria cultured for 25 days; Supplemental Figure 1B), along with diminished FSP1 and vimentin expression (Supplemental Figure 1, E and H, respectively). In contrast, $100 \mathrm{ng} / \mathrm{ml}$ CTGF delivery rescued the calvarial suture from undergoing synostosis, along with restored FSP1 and vimentin expression (Supplemental Figure 1, C, F, and I). The CTGF-rescued calvarial suture showed patency, in contrast to the virtual closure of the CTGF-free suture by $\mu \mathrm{CT}$ (Supplemental Figure $1, \mathrm{~J}$ and $\mathrm{K}$ ), which was confirmed by quantitative data showing that CTGF delivery yielded significantly greater suture width than without CTGF (Supplemental Figure 1L). Tn-C content, as assessed by ELISA, was significantly greater in CTGF-rescued than in CTGF-free calvarial sutures (Supplemental Figure 1M), further suggesting that CTGF prompted fibrogenesis.

Furthermore, cells isolated from native, patent calvarial sutures, upon 100-ng/ml CTGF treatment by P7, readily differentiated into fibroblast-like cells highly positive to Masson trichrome staining (Supplemental Figure 2A), which suggests that multipotent mesenchymal cells in either appendicular bone marrow (as 
described above) or calvaria are capable of fibroblastic differentiation. In contrast, isolated calvarial suture cells without CTGF treatment continued to assume MSC morphology and synthesized little collagen (Supplemental Figure 2E). Interestingly, cells isolated from P7 calvarial suture at the threshold of synostosis (i.e., P25) readily differentiated into osteoblasts under osteogenic stimulation (see Methods) with or without CTGF (Supplemental Figure 2, B and C), in comparison to isolated cells without osteogenic stimulation (Supplemental Figure 2F). Also, isolated calvarial cells underwent adipogenic differentiation under permissive conditions (see Methods and Supplemental Figure 2D), in comparison to isolated cells without adipogenic stimulation (Supplemental Figure 2G). These findings suggest that calvarial suture is composed of multipotent mesenchymal cells that readily differentiate into fibroblastic cells and undergo fibrogenesis upon CTGF stimulation, in addition to differentiation into other mesenchymal lineages. Fibroblastic differentiation of calvarial mesenchymal cells provides the motivation for our in vivo approach for microencapsulated delivery of CTGF to promote fibrogenesis in connective tissue healing.

\section{Discussion}

MSCs that are isolated as mononucleated and adherent cells from bone marrow and other connective tissue sources are typically heterogeneous $(8,9,38)$. The present data show that approximately $67 \%$ of the established clones from randomly plated MSC clones differentiated into each and all of osteogenic, chondrogenic, adipogenic, and fibroblastic lineages in chemically defined media. In comparison, 2 clonal progenies (approximately 11\%) differentiated into fibroblasts, osteoblasts, and chondrocytes, but not adipocytes, whereas the remaining 4 clonal progenies (approximately $22 \%$ ) failed to differentiate into any of these cells. Although fibroblasts in heterogeneous MSC culture may have been stimulated to proliferate by CTGF, single MSC progenies were indeed capable of differentiating into fibroblastic cells. These cloning data further substantiate the innate capacity of MSCs to differentiate into common connective tissue phenotypes, including bone, cartilage, and adipose. Remarkably, our MSC culture contained approximately $22 \%$ of clonal progenies of bone marrow mononucleated, adherent cells incapable of differentiation into any of fibroblastic, osteogenic, chondrogenic, or adipogenic lineages, which suggests that these cells are likely terminally differentiated cells, such as fibroblasts, osteoblasts, and other nonhematopoietic cells. Interestingly, our finding that approximately $11 \%$ of randomly plated bone marrow mononucleated, adherent cells differentiated into some, but not all, of the fibroblastic, osteogenic, chondrogenic, and adipogenic lineages was further indicative of the heterogeneity of typical MSC culture. Limiting dilution, a common approach for cell cloning, was adopted here to derive MSC clonal progenies instead of cell sorting, due to a lack of unique cell surface epitopes for both MSCs and fibroblasts $(5,8)$. Thus, approximately $28 \%$ of randomly plated bone marrow adherent cells, or approximately $67 \%$ of clonal progenies (present study), are capable of differentiating into each and all of fibroblastic, osteogenic, chondrogenic, and adipogenic cells. Accordingly, fibroblast colony-forming units initially isolated as nonhematopoietic, heterogeneous cells of bone marrow by Friedenstein et al. (10), similar to most bone marrow MSC culture today, are indeed heterogeneous, but nonetheless contain multipotent stem/progenitor cells. Following cloning, we elected to characterize $\alpha$-SMA phenotype of MSCs as a heterogeneous population, rather than MSC clonal progenies, primarily because MSCs participate in wound healing rarely as purified cells, but rather by interactions with vascular cells and immune competent cells (7-9). Thus, heterogeneous bone marrow MSCs appear to be relevant to tissue regeneration in vivo, whereas clonal progenies of MSCs may be of interest to particular disease models that are yet to be understood $(7-9,30)$.

Our findings showed derivation of fibroblasts from multipotent mesenchymal cells. A mesenchymal origin of fibroblasts is complimentary to EMT $(14-16,39)$. EMT has been identified in fibrosis of organs, including the heart, kidneys, and lungs. EMT explains the origin of fibroblasts in organ fibrosis in which fibroblasts are stromal cells, but not in tissues in which fibroblasts are parenchymal cells, given the absence of either epithelial or endothelial cells in normal tendons or ligaments. Even in organ fibrosis, EMT does not account for all the observed fibroblasts $(14,17)$. Also, it has been speculated that EMT applies to pathological conditions in which basement membrane between fibroblastic stroma and epithelial/endothelial cells is broken $(16,17)$. It is conceivable, but yet to be tested, that parenchymal fibroblasts and/or unaccounted-for fibroblasts in organ fibrosis are derived from MSCs that reside in situ in connective tissue or are recruited systemically from bone marrow. Additional experiments are warranted to lineage-trace fibroblasts and/or MSCs in vivo.

CTGF-derived fibroblastic cells appear to be a stable cell population with diminished ability to differentiate into other mesenchymal lineages, including osteoblasts, chondrocytes, and adipocytes. Our ex vivo and in vivo data suggest that CTGF favors fibrogenesis, rather than osteogenesis. Previously, fibroblasts have been proposed to derive from 3 sources: EMT, tissue-resident progenitors, and bone marrow $(3,24,40)$. Whereas EMT has gained experimental support, only sporadic observations have been made for a putative mesenchymal origin of fibroblasts $(41,42)$. The mesenchymal origin of fibroblasts is complementary to a recent discovery of multipotent stem/progenitor cells in tendons (20). Interestingly, the stem/progenitor cells of bone marrow origin from which fibroblasts were derived in the present study were not $\mathrm{CD} 34^{+}$and $\alpha$-SMA ${ }^{+}$fibrocytes, which have previously been postulated to migrate from bone marrow to cancer stroma or peripheral wounds (23-25). Rather, CD34mesenchymal cells in the present study differed substantially from $\mathrm{CD} 34^{+}$cells of hematopoietic lineage $(43,44)$. Whether $\mathrm{CD}^{+} 4^{+}$and $\mathrm{CD} 34^{-}$progenitors in bone marrow both give rise to $\mathrm{FSP} 1^{+}$, vimen$\operatorname{tin}^{+}$, and Col-I fibroblasts warrants additional investigation.

Fibrosis and scarring is a universal response to traumatic insult, yet an insufficiently studied challenge in tissue regeneration. Fibroblast contraction of granular tissue is a process of normal wound healing $(3,24)$. In pathological wound healing, the activation of fibroblasts by acquiring $\alpha$-SMA phenotype and excessive contractility are among the factors responsible for organ fibrosis or aberrant dermal scarring $(3,40,45)$, including keloids and hypertrophic scars, for which there is currently no satisfactory therapy $(45,46)$. An important distinction between fibrogenesis and fibrosis appears to be $\alpha$-SMA expression. Despite their presence in the early stage of wound, $\alpha-\mathrm{SMA}^{+}$myofibroblasts disappear, presumably by apoptosis, as wound healing progresses $(1,2,4,5)$. MSCs and mesenchymally derived fibroblasts in our study were overwhelmingly $\alpha$-SMA-. Our data suggest that fibroblastic differentiation, fibrogenesis, and fibrosis appear to be 3 distinct processes. CTGF prompted the differentiation of MSCs into FSP1 $1^{+}$, vimentin ${ }^{+}$, Col- $\mathrm{I}^{+}$, and $\alpha$-SMA- fibroblasts, but not $\alpha$-SMA ${ }^{+}$myofibroblasts, which suggests that CTGF-derived 
fibroblasts participate in normal wound healing. TGF- $\beta 1$ stimulation subsequent to CTGF treatment induced differentiation of $\alpha$-SMA- fibroblasts into $\alpha$-SMA ${ }^{+}$myofibroblasts, which are relevant to insults such as invasive tumor (cancer stroma), organ fibrosis, and aberrant dermal healing.

How stem/progenitor cells select their fate between fibrogenesis and osteogenesis is poorly understood in a cascade of seemingly unrelated disorders, including cardiac valve calcification, vascular arthrosclerosis, craniosynostosis, calcified dermal healing, mineralization around implanted medical devices, and tendinopathy (31-33). CTGF has been shown to favor fibrogenesis in connective tissue healing, as in the present craniosynostosis model, over ectopic mineralization. CTGF-deficient mice develop skeletal dysmorphism and impaired extracellular matrix production and turnover (47). CTGF expression is attenuated in both BMP-9- and Wnt3Ainduced osteogenic differentiation (48). The existing concept for surgical correction of craniosynostosis is that aberrantly high osteogenesis is at fault, leading to the current surgical practice of craniotomy by removing synostosed calvarial sutures. The present findings suggest that localized surgery along with CTGF delivery may substitute for craniotomy as a minimally invasive approach. Furthermore, mesenchymally derived fibroblasts may act as repair cells for ligament and tendon injuries, which affect millions of new patients per year, but for which few effective therapies exist $(18,19)$. An important recent finding shows that tendon harbors multipotent stem/progenitor cells that differentiate into typical mesenchymal lineages, including adipose, bone, and cartilage cells (20). However, it is difficult for tendon-derived stem/progenitor cells to act as an autologous repair cell source without scarifying normal tendon. In contrast, bone marrow MSCs can be readily aspirated as a clinically accepted procedure and differentiated into fibroblasts that may be clinically relevant for tendon and ligament regeneration. Taken together, the present findings may have implications in our understanding of development, tissue regeneration, dermal healing, organ fibrosis, and cancer stroma.

\section{Methods}

Cell isolation and expansion. Human MSCs were isolated from fresh whole bone marrow samples of 2 anonymous adult donors (age range, 20-25 years; AllCells) and were exempt from IRB approval. Nonhematopoietic, mononucleated and adherent cells were purified by centrifugation through a density gradient (Ficoll-Paque), as we previously described $(12,27)$ and according to the manufacturer's protocols (RosetteSep; StemCell Technologies), to remove hematopoietic cells. Briefly, bone marrow was transferred to a $50-\mathrm{ml}$ tube, followed by addition of $750 \mathrm{ml}$ RosetteSep and incubation for $20 \mathrm{~min}$ utes. Then $15 \mathrm{ml}$ PBS in 2\% FBS and 1-mM EDTA was added, to a total volume of approximately $30 \mathrm{ml}$. The sample was layered on $15-\mathrm{ml}$ Ficoll-Paque and centrifuged for 25 minutes at $300 \mathrm{~g}$. The entire layer of enriched cells was removed from Ficoll-Paque interface. Collected cells were counted and tested with trypan blue, plated at $0.5-1 \times 10^{6}$ cells per $100-\mathrm{mm}$ dish, and allowed to attach for approximately 5 days, followed by regular medium change every 2-3 days. At $80-90 \%$ confluence, cells were trypsinized, centrifuged, resuspended in growth medium as passage 1 cells, and incubated in $5 \% \mathrm{CO}_{2}$ at $37^{\circ} \mathrm{C}$, with fresh medium changes every 3-4 days. Growth medium was composed of DMEM-low glucose (DMEM-LG; Sigma-Aldrich), $1 \%$ antibiotic ( $1 \times$ antibiotic-antimycotic, including $10 \mathrm{U} / 1$ penicillin $\mathrm{G}$ sodium, $10 \mathrm{mg} / \mathrm{ml}$ streptomycin sulfate, and $0.25 \mu \mathrm{g} / \mathrm{ml}$ amphotericin B; Gibco, Invitrogen), and 10\% FBS (Atlanta Biologicals). To isolate calvarial mesenchymal cells, calvarial suture samples with small adjacent bone marrow were carefully dissected from P7 rat calvaria using surgical scissors. The overlying periosteum and the underlying dura mater were removed under dissection microscope. The isolated samples were lysed using $2-\mathrm{mg} / \mathrm{ml}$ collagenase for 1 hour at $37^{\circ} \mathrm{C}$ and filtered with a tissue strainer (Fisher). After centrifugation, the collected cells were counted and tested with trypan blue, plated at 20,000-30,000 cells per 6-well plate, and allowed to attach for approximately 5 days, followed by medium change every 2-3 days. Passage 1 or 2 calvarial cells were induced for osteogenic, chondrogenic, adipogenic, or fibroblastic differentiation, as described below.

Treatment with CTGF, Col-I and Tn-C synthesis, and real-time PCR. The effective CTGF doses for fibroblastic differentiation were determined. Passage 3 or 4 MSCs were culture expanded in monolayer (about 5,000 cells/well) in 12 -well plates. At $80 \%-90 \%$ confluence, MSCs were treated with 0, 10, 50, or $100 \mathrm{ng} / \mathrm{ml}$ recombinant human CTGF (BioVendor) and $50 \mu \mathrm{g} / \mathrm{ml}$ ascorbic acid (Sigma-Aldrich), with conditioned medium change every third day. Col-I matrix was lysed using $0.5 \mathrm{M}$ acetic acid and quantified by ELISA (see below). The dose of $100 \mathrm{ng} / \mathrm{ml}$ CTGF was selected for fibroblastic differentiation, since it induced substantial collagen synthesis by Goldner trichrome staining (Figure 1E). Col-I and Tn-C synthesis was quantified by ELISA with commercial kits (Chondrex and IBL-America). Total RNA was isolated using TRIzOL and sample incubation for 5 minutes at room temperature. A total of $0.2 \mathrm{ml}$ chloroform per $1 \mathrm{ml} \mathrm{TRIzOL}$ was added, followed by incubation for 3 minutes. After centrifugation at $12,000 \mathrm{~g}$ and $4^{\circ} \mathrm{C}$ for 15 minutes, the upper phase was transferred into a new tube with $500 \mu \mathrm{l}$ isopropanol. After 10 minutes of incubation and 10 minutes of centrifugation at $12,000 \mathrm{~g}$, the supernatant was discarded. The pellet was washed with $1 \mathrm{ml} 75 \%$ ethanol and dried for 5-10 minutes. RNA samples were eluted in $50 \mu \mathrm{l}$ RNase-free water, assessed for concentration and purity at 260 and $280 \mathrm{~nm}$, and stored at $-80^{\circ} \mathrm{C}$ prior to reverse transcription. All RNA samples were reverse transcribed (Applied Biosystems). For mRNA quantification, real-time quantitative $\mathrm{PCR}$ reactions with the cDNA samples were performed using 7300 Real-Time PCR System TaqMan gene expression assays (Applied Biosystems). Commercial primers and probes for human Col-I, Col-III, Tn-C, fibronectin, MMP-1, FSP1, and vimentin were used $(49,50)$. Col-II and osteocalcin were selected as chondrogenic and osteogenic differentiation markers, respectively. CD29, CD44, CD105, CD106, CD117, BMPR1A, and sca1 were selected as MSC surface epitopes $(12,27)$. GAPDH was used as housekeeping gene.

Osteogenic, chondrogenic, and adipogenic differentiation. Table 2 shows induction factors and assays for fibrogenic, osteogenic, chondrogenic, and adipogenic differentiation of MSCs. Osteogenic differentiation medium contained $100 \mathrm{nM}$ dexamethasone, $10 \mathrm{mM} \beta$-glycerophosphate, and $0.05 \mathrm{mM}$ ascorbic acid-2-phosphate (Sigma-Aldrich), as we described previously $(12,51)$. Chondrogenic medium was supplemented with $10 \mathrm{ng} / \mathrm{ml}$ TGF- $\beta 3$ (R\&D Systems; refs. 12, 51). Adipogenic differentiation medium consisted of basal medium supplemented with $0.5 \mu \mathrm{M}$ dexamethasone, $0.5 \mu \mathrm{M}$ isobutyl methylxanthine, and $50 \mu \mathrm{M}$ indomethacin, as we described previously $(35,52)$. von Kossa staining and calcium assay was performed to evaluate osteogenic differentiation, whereas safranin O straining and glycosaminoglycan assays were performed to evaluate chondrogenic differentiation (Blyscan). Oil Red O staining (Sigma-Aldrich) was used to verify adipogenesis (lipid formation).

Cloning. MSC clones were established by limiting dilution. Briefly, passage 0 MSCs were suspended at a concentration of 1 cell per $200 \mu \mathrm{l}$ and plated in 96 wells with $200 \mu \mathrm{l}$ medium per well. After 24 hours, wells with a single cell were selected for culture in DMEM-LG with $1 \%$ antibiotic and $10 \% \mathrm{FBS}$ at $37^{\circ} \mathrm{C}$ and $5 \% \mathrm{CO}_{2}$, with medium change twice per week. After 3-4 weeks, single cell-derived clones were treated with $0.25 \%$ trypsin-EDTA and seeded into 24-well plates to expand into clonal progenies. Clonal progenies were transferred to 6-well plates upon confluence of approximately $80 \%$ and maintained with medium change every $3-4$ days. 


\section{Table 2}

Multilineage differentiation of MSCs

$\begin{array}{lll}\text { Lineage } & \text { Induction supplements } & \text { Assay } \\ \text { Fibrogenic } & 100 \mathrm{ng} / \mathrm{ml} \text { CTGF, } & \text { Masson trichrome } \\ & 50 \mu \mathrm{g} / \mathrm{ml} \text { ascorbic acids } & \\ \text { Chondrogenic } & 10 \mathrm{ng} / \mathrm{ml} \mathrm{TGF- \beta 3} & \text { Safranin 0 } \\ \text { Adipogenic } & 0.5 \mu \mathrm{M} \text { dexamethasone, } & \text { Oil Red 0 } \\ & 0.5 \mu \mathrm{M} \text { isobutyl methylxanthine, } & \\ & 50 \mu \mathrm{M} \text { indomethacin } & \\ \text { Osteogenic } & 100 \mathrm{nM} \text { dexamethasone, } & \text { Alizarin red } \\ & 10 \mathrm{mM} \text {-glycerophosphate, } & \\ & 0.05 \mathrm{mM} \text { ascorbic acid-2-phosphate }\end{array}$

Myofibroblastic differentiation of MSC-derived fibroblasts and collagen gel contraction assay. MSC-derived fibroblasts were subjected to $5 \mathrm{ng} / \mathrm{ml} \mathrm{recom-}$ binant human TGF- $\beta 1$ (R\&D Systems). Native MSCs were treated with TGF- $\beta 1$ as control. Cloned cells were not used for this experiment, primarily because MSCs participate in wound healing rarely as purified cells, but rather by interactions with vascular and immunocompetent cells (7-9). The expression of $\alpha$-SMA (Abcam) was evaluated by immunofluorescence, with rhodamine-phalloidin staining for microfilaments and DAPI for nuclei (Invitrogen). The number of $\alpha$-SMA ${ }^{+}$cells was quantified by flow cytometry. Briefly, cells were trypsinized, counted, resuspended into $1 \times 10^{6}$ cells/tube, and fixed in $0.01 \%$ formaldehyde. After washing in $0.1 \%$ triton, the cells were incubated with primary $\alpha$-SMA antibody (Abcam) for 30 minutes. After $400 \mathrm{~g}$ centrifugation for 5 minutes, the cells were incubated with fluorochrome-labeled secondary antibody (Abcam) for 30 minutes in the dark and resuspended in PBS supplemented with 3\% BSA and $1 \%$ sodium azide. The samples were then analyzed using a cell sorter (FACSAria II; BD Biosciences).

Neutralized Col-I ( $2 \mathrm{mg} / \mathrm{ml}$; R\&D Systems) was mixed thoroughly with MSCs or MSC-derived fibroblasts with or without TGF- $\beta 1$ at $1 \times 10^{6}$ cells $/ \mathrm{ml}$. Cell-populated collagen solution in 24-well plates was incubated for 1 hour at $37^{\circ} \mathrm{C}$ to induce gelation. After 48 hours of incubation, cell-populated collagen lattices were physically detached and allowed to float freely in medium. Up to the tested 7 days, the diameters of collagen lattices were measured on digital images.

Ex vivo modulation of calvarial morphogenesis by CTGF. Calvarial explants, including frontal and parietal bones with intervening interfrontal, coronal, and sagittal sutures, were harvested with intact dura mater from P10 male Sprague-Dawley rats (Harlan) following Columbia University IACUC approval. Isolated calvaria were placed in 12 wells with serum-free medium supplemented with 0 or $50 \mathrm{ng} / \mathrm{ml}$ recombinant human CTGF (37), with medium change every 2 days. The CTGF concentration was determined in a pilot experiment (data not shown). At 5-25 days following CTGF treatment, Tn-C contents in supernatant were assayed using ELISA. Explantcultured calvarial sutures were sectioned sagittally for $\mathrm{H} \& \mathrm{E}$ staining and immunohistochemistry. Calvarial sutures were scanned with $\mu$ CT (vivaCT 40; Scanco) to measure suture width.

Preparation ofCTGF-encapsulated microspheres. Poly-D-L-lactic-co-glycolic acid (PLGA) microspheres were fabricated by double emulsion, as we described previously $(35,52)$. Briefly, a total of $250 \mathrm{mg}$ PLGA was dissolved into $1 \mathrm{ml}$ dichloromethane. Recombinant human CTGF $(10 \mu \mathrm{g})$ was diluted to $50 \mu \mathrm{l}$ and added to the PLGA solution, forming a mixture (primary emulsion) that was emulsified for 1 minute (water-in-oil). The primary emulsion was then added to $2 \mathrm{ml}$ 1\% polyvinyl alcohol (PVA; 30,000-70,000 MW), followed by 1 minute mixing ([water-in-oil]-in-water). Upon addition of $100 \mathrm{ml} \mathrm{PVA}$, the mixture was stirred for 1 minute. A total of $100 \mathrm{ml} 2 \%$ isopropanol was added to the final emulsion and continuously stirred for 2 hours to remove the solvent. Control microspheres (empty and without CTGF) were fabricated in the same fashion by replacing CTGF with $50 \mu$ l distilled water.

In vivo calvarial regeneration by control-released CTGF. Sprague-Dawley rats develop native ectopic mineralization or synostosis in the interfrontal suture by approximately P25 $(36,37,53,54)$. After receiving Columbia University IACUC approval, we anesthetized rats with $1 \%-5 \%$ isoflurane. A $2 \times 4 \mathrm{~mm}$ defect was created by resecting the posterior interfrontal suture using a dental bur with PBS irrigation and care not to damage the underlying dura mater (36). Collagen sponge (Integra LifeSciences) containing $10 \mathrm{mg}$ CTGFor PBS-encapsulated microspheres was implanted in the surgically created defect. At 4 postoperative weeks, tissues were harvested and analyzed. The harvested tissues were sectioned every $4 \mu \mathrm{m}$ for histology and for FSP1 and vimentin immunohistochemistry (Santa Cruz Biotechnology).

Statistics. After confirmation of normal data distribution, 1-way ANOVA with post-hoc Bonferroni tests were used, with a $P$ value less than 0.05 considered significant.

\section{Acknowledgments}

We are indebted to D. Prockop and R. Tuan for their valuable comments. We thank E. Guo and E. Chen for guidance in and access to $\mu \mathrm{CT}$ scanning of ex vivo calvarial samples and F. Guo and K. Hua for technical and administrative support. The work was supported in part by NIH grant 5RC2DE020767 from the National Institute of Dental and Craniofacial Research to J.J. Mao.

Received for publication April 5, 2010, and accepted in revised form June 16, 2010.

Address correspondence to: Jeremy J. Mao, Columbia University Medical Center, 630 W. 168 St., PH7E CDM, New York, New York 10032, USA. Phone: 212.305.4475; Fax: 212.342.0199; E-mail: jmao@columbia.edu.
1. Bhowmick NA, et al. TGF-beta signaling in fibroblasts modulates the oncogenic potential of adjacent epithelia. Science. 2004;303(5659):848-851.

2. Bhowmick NA, Neilson EG, Moses HL. Stromal fibroblasts in cancer initiation and progression. Nature. 2004;432(7015):332-337.

3. Darby IA, Hewitson TD. Fibroblast differentiation in wound healing and fibrosis. Int Rev Cytol. 2007;257:143-179.

4. Karnoub AE, et al. Mesenchymal stem cells within tumour stroma promote breast cancer metastasis. Nature. 2007;449(7162):557-563.

5. Sorrell JM, Caplan AI. Fibroblasts-a diverse population at the center of it all. Int Rev Cell Mol Biol. 2009;276:161-214.

6. Chang HY, et al. Diversity, topographic differentia- tion, and positional memory in human fibroblasts. Proc Natl Acad Sci U S A. 2002;99(20):12877-12882.

7. Bianco P, Robey PG, Simmons PJ. Mesenchymal stem cells: revisiting history, concepts, and assays. Cell Stem Cell. 2008;2(4):313-319.

8. Caplan AI. Mesenchymal stem cells. J Orthop Res. 1991;9(5):641-650.

9. Prockop DJ. Marrow stromal cells as stem cells for nonhematopoietic tissues. Science. 1997; 276(5309):71-74.

10. Friedenstein AJ, et al. Precursors for fibroblasts in different populations of hematopoietic cells as detected by the in vitro colony assay method. Exp Hematol. 1974;2(2):83-92.

11. Owen M, Friedenstein AJ. Stromal stem cells: marrow-derived osteogenic precursors. Ciba Found
Symp. 1988;136:42-60.

12. Marion NW, Mao JJ. Mesenchymal stem cells and tissue engineering. Methods Enzymol. 2006; 420:339-361.

13. Herzlinger $D$. Renal interstitial fibrosis: remembrance of things past? JClin Invest. 2002;110(3):305-306.

14. Kalluri R, Neilson EG. Epithelial-mesenchymal transition and its implications for fibrosis. J Clin Invest. 2003;112(12):1776-1784.

15. Zeisberg M, Kalluri R. The role of epithelial-tomesenchymal transition in renal fibrosis. J Mol Med. 2004;82(3):175-181.

16. Iwano M, Plieth D, Danoff TM, Xue C, Okada H, Neilson EG. Evidence that fibroblasts derive from epithelium during tissue fibrosis. J Clin Invest. 2002;110(3):341-350. 
17. Zeisberg M, Neilson EG. Biomarkers for epithelial-mesenchymal transitions. J Clin Invest. 2009;119(6):1429-1437.

18. Van Eijk F, et al. Tissue engineering of ligaments: a comparison of bone marrow stromal cells, anterior cruciate ligament, and skin fibroblasts as cell source. Tissue Eng. 2004;10(5-6):893-903.

19. Woo S-Y, et al. Anatomy, biology, and biomechanics of tendon and ligament. In: Buckwalter JA, Einhon TA, Simon SR, eds. Orthopaedic Basic Science. 2nd ed. Rosemont, Illinois, USA: American Academy of Orthopaedic Surgeons; 2001:582-616.

20. Bi Y, et al. Identification of tendon stem/progenitor cells and the role of the extracellular matrix in their niche. Nat Med. 2007;13(10):1219-1227.

21. McAnulty RJ. Fibroblasts and myofibroblasts: their source, function and role in disease. Int J Biochem Cell Biol. 2007;39(4):666-671.

22. Dominici $M$, et al. Minimal criteria for defining multipotent mesenchymal stromal cells. The International Society for Cellular Therapy position statement. Cytotherapy. 2006;8(4):315-317.

23. Bellini A, Mattoli S. The role of the fibrocyte, a bone marrow-derived mesenchymal progenitor, in reactive and reparative fibroses. Lab Invest. 2007; 87(9):858-870.

24. Kisseleva T, Brenner DA. Mechanisms of fibrogenesis. Exp Biol Med (Maywood). 2008;233(2):109-122.

25. Wynn RF, et al. A small proportion of mesenchymal stem cells strongly expresses functionally active CXCR4 receptor capable of promoting migration to bone marrow. Blood. 2004;104(9):2643-2645.

26. Perbal B. CCN proteins: multifunctional signalling regulators. Lancet. 2004;363(9402):62-64.

27. Alhadlaq A, Mao JJ. Mesenchymal stem cells: isolation and therapeutics. Stem Cells Dev. 2004; 13(4):436-448

28. Gressner OA, Gressner AM. Connective tissue growth factor: a fibrogenic master switch in fibrotic liver diseases. Liver Int. 2008;28(8):1065-1079.

29. Shi-Wen X, Leask A, Abraham D. Regulation and function of connective tissue growth factor/CCN2 in tissue repair, scarring and fibrosis. Cytokine Growth Factor Rev. 2008;19(2):133-144.

30. Yang R, Chen M, Lee CH, Yoon R, Lal S. Clones of ectopic stem cells in the regeneration of muscle defects in vivo. PLoS One. In press.

31. Giachelli CM. Ectopic calcification: gathering hard facts about soft tissue mineralization. Am J Pathol. 1999;154(3):671-675.

32. Kirsch T. Determinants of pathologic mineralization. Crit Rev Eukaryot Gene Expr. 2008;18(1):1-9.

33. Persy V, D'Haese P. Vascular calcification and bone disease: the calcification paradox. Trends Mol Med. 2009;15(9):405-416.

34. Moioli EK, Clark PA, Xin X, Lal S, Mao JJ. Matrices and scaffolds for drug delivery in dental, oral and craniofacial tissue engineering. Adv Drug Deliv Rev. 2007;59(4-5):308-324.

35. Moioli EK, Hong L, Guardado J, Clark PA, Mao JJ. Sustained release of TGFbeta3 from PLGA microspheres and its effect on early osteogenic differentiation of human mesenchymal stem cells. Tissue Eng. 2006;12(3):537-546.

36. Moioli EK, Clark PA, Sumner DR, Mao JJ. Autologous stem cell regeneration in craniosynostosis. Bone. 2008;42(2):332-340.

37. Opperman LA, Passarelli RW, Morgan EP, Reintjes $\mathrm{M}$, Ogle RC. Cranial sutures require tissue interactions with dura mater to resist osseous obliteration in vitro. J Bone Miner Res. 1995;10(12):1978-1987.

38. Battula VL, et al. Isolation of functionally distinct mesenchymal stem cell subsets using antibodies against CD56, CD271, and mesenchymal stem cell antigen-1. Haematologica. 2009;94(2):173-184.

39. Neilson EG, Plieth D, Venkov C. Epithelialmesenchymal transitions and the intersecting cell fate of fibroblasts and metastatic cancer cells. Trans Am Clin Climatol Assoc. 2003;114:87-100.

40. Leask A. Targeting the TGFbeta, endothelin-1 and CCN2 axis to combat fibrosis in scleroderma. Cell Signal. 2008;20(8):1409-1414.

41. Altman GH, et al. Cell differentiation by mechanical stress. FASEB J. 2002;16(2):270-272.

42. Moreau JE, Chen J, Horan RL, Kaplan DL, Altman $\mathrm{GH}$. Sequential growth factor application in bone marrow stromal cell ligament engineering. Tissue Eng. 2005;11(11-12):1887-1897.

43. Moioli EK, et al. Synergistic actions of hematopoietic and mesenchymal stem/progenitor cells in vascularizing bioengineered tissues. PLoS One. 2008;3(12):e3922.

44. Weissman IL, Shizuru JA. The origins of the identification and isolation of hematopoietic stem cells, and their capability to induce donor-specific transplantation tolerance and treat autoimmune diseases. Blood. 2008;112(9):3543-3553.

45. Gurtner GC, Werner S, Barrandon Y, Longaker MT. Wound repair and regeneration. Nature. 2008;453(7193):314-321.

46. Hinz B. Formation and function of the myofibroblast during tissue repair. J Invest Dermatol. 2007;127(3):526-537.

47. Ivkovic $S$, et al. Connective tissue growth factor coordinates chondrogenesis and angiogenesis during skeletal development. Development. 2003;130(12):2779-2791.

48. Luo Q, et al. Connective tissue growth factor (CTGF) is regulated by Wnt and bone morphogenetic proteins signaling in osteoblast differentiation of mesenchymal stem cells. J Biol Chem. 2004;279(53):55958-55968.

49. Hankemeier $S$, et al. Modulation of proliferation and differentiation of human bone marrow stromal cells by fibroblast growth factor 2: potential implications for tissue engineering of tendons and ligaments. Tissue Eng. 2005;11(1-2):41-49.

50. Moreau JE, et al. Growth factor induced fibroblast differentiation from human bone marrow stromal cells in vitro. J Orthop Res. 2005;23(1):164-174.

51. Alhadlaq A, et al. Adult stem cell driven genesis of human-shaped articular condyle. Ann Biomed Eng. 2004;32(7):911-923.

52. Stosich MS, Bastian B, Marion NW, Clark PA, Reilly G, Mao JJ. Vascularized adipose tissue grafts from human mesenchymal stem cells with bioactive cues and microchannel conduits. Tissue Eng. 2007;13(12):2881-2890.

53. Moss ML. Growth of the calvaria in the rat; the determination of osseous morphology. Am J Anat. 1954;94(3):333-361.

54 . Roth DA, et al. Immunolocalization of transforming growth factor beta 1 , beta 2 , and beta 3 and insulin-like growth factor I in premature cranial suture fusion. Plast Reconstr Surg. 1997;99(2):300-309. 